\title{
Terapia fonológica a partir da estimulação de sons isolados e em conjunto
}

\author{
Phonological therapy based on the stimulation of single sounds \\ and groups of sounds
}

\author{
Karina Carlesso Pagliarin¹, Ana Rita Brancalioni², Márcia Keske-Soares ${ }^{3}$
}

\begin{abstract}
RESUMO
Objetivo: Verificar se a estimulação de sons em conjunto resulta em maiores mudanças fonológicas do que a estimulação de sons isoladamente e analisar a ocorrência de generalização para dentro de uma classe e para outras classes de sons. Métodos: A amostra foi composta por dez sujeitos com desvio fonológico, com idades entre 4 anos e 8 meses a 7 anos e 3 meses, distribuídos em dois grupos. O Grupo 1 (G1) foi tratado pelo modelo de Oposições Múltiplas e o Grupo 2 (G2) pelo modelo ABAB-Retirada e Provas Múltiplas. O tratamento do G1 constou da estimulação do contraste de vários sons apresentados em conjunto enquanto que o tratamento do G2 envolveu a estimulação de apenas um som-alvo por ciclo de tratamento. Foi analisado o número de sons ausentes e a ocorrência de generalização para dentro de uma classe e para outras classes de sons pré e pós-tratamento. Os dados foram analisados estatisticamente; também foi realizada análise descritiva. Resultados: O G1 adquiriu maior número de sons que o G2; entretanto não houve diferença significativa. Em relação à generalização dentro da classe de sons, o G1 apresentou maiores mudanças, enquanto a generalização para outras classes foi maior para o G2; entretanto, não houve diferença estatística em nenhum desses resultados. Conclusão: Ambos os métodos favoreceram mudanças no sistema fonológico das crianças estudadas, levando à aquisição de fonemas e a generalizações. Não há diferença entre eles na efetividade do tratamento.
\end{abstract}

Descritores: Distúrbios da fala; Fala; Fonoterapia; Generalização da resposta; Som; Criança

\section{INTRODUÇÃO}

A busca por tratamentos mais efetivos e eficazes para o desvio fonológico deu origem a diversos modelos terapêuticos que se diferenciam quanto a seus pressupostos teóricos, sendo que atualmente, existem mais de dez. Dentre eles destacam-se o Modelo de Pares Mínimos ${ }^{(1)}$, de Oposições Máximas ${ }^{(2,3)}$, de Oposições Múltiplas ${ }^{(4)}$, ABAB-Retirada e Provas Múltiplas ${ }^{(5)}$, Metaphon $^{(6)}$, Ciclos Modificado ${ }^{(7)}$ e a Intervenção Metafonológica $^{(8)}$. Este último ainda não foi aplicado em crianças falantes do português brasileiro.

Trabalho realizado no Centro de Estudos de Linguagem e Fala, Universidade Federal de Santa Maria - UFSM - Santa Maria (RS), Brasil.

(1) Programa de Pós-graduação (Doutorado) em Psicologia (área de concentração: Cognição Humana), Pontifícia Universidade Católica do Rio Grande do Sul - PUCRS - Porto Alegre (RS), Brasil.

(2) Programa de Pós-graduação (Doutorado) em Distúrbios da Comunicação Humana, Universidade Federal de Santa Mara - UFSM - Santa Maria (RS), Brasil.

(3) Curso de Fonoaudiologia e Programa de Pós-graduação em Distúrbios da Comunicação Humana, Universidade Federal de Santa Maria - UFSM Santa Maria (RS), Brasil.

Endereço para correspondência: Karina Carlesso Pagliarin. R. Coronel Scherer, 09, São Pedro do Sul (RS), Brasil, CEP: 97400-000. E-mail: karinap_fono@yahoo.com.br

Recebido em: 29/7/2010; Aceito em: 10/1/2011
Devido à existência de tantos modelos de terapia fonológica, torna-se importante a realização de pesquisas com o intuito de compará-los, o que contribuirá para a escolha do método mais adequado para implementação na clínica fonoaudiológica. Para selecionar o tratamento mais adequado, o clínico deve levar em consideração o sistema fonológico da criança, a fim de selecionar tanto o modelo terapêutico como os sons-alvo.

Estudos recentes têm aplicado modelos terapêuticos e analisado os diferentes tipos de generalização ${ }^{(9-11)}$, enquanto outros tem comparado as diversas abordagens de tratamento do desvio fonológico e verificado sua eficácia ${ }^{(12-14)}$. Entretanto, nenhum estudo comparou modelos considerando a quantidade de alvos selecionados para terapia, o que torna esta pesquisa importante, pois acredita-se que quanto mais sons estimulados, mais rápido será a aquisição e maior a generalização ${ }^{(14)}$.

Os modelos ABAB-Retirada e Provas Múltiplas e o de Oposições Múltiplas, diferem quanto à estrutura da sessão e, principalmente, quanto à quantidade de alvos selecionados para a terapia. O primeiro consta da estimulação de apenas um som-alvo por ciclo de tratamento (nove sessões), e o último do contraste de vários sons apresentados em conjunto (simultaneamente) durante todas as sessões que compõem 
o ciclo de terapia (cinco sessões ou mais). Trabalhar sons simultaneamente pode tornar a terapia fonológica mais efetiva uma vez que enfoca contrastes múltiplos, necessários à aquisição da fonologia. Além disso, a estimulação de várias dificuldades, simultaneamente, pode tornar processo terapêutico mais rápido.

Este estudo teve como objetivos verificar se a estimulação de sons em conjunto resulta em maiores mudanças fonológicas do que a estimulação de um único som e analisar a ocorrência de generalização para dentro de uma classe e para outras classes de sons.

\section{MÉTODOS}

Para a realização desta pesquisa foi utilizado o banco de dados do Centro de Estudos de Linguagem e Fala sediado no departamento de fonoaudiologia da Universidade Federal de Santa Maria (UFSM). A amostra foi constituída por dez sujeitos com desvio fonológico, com idades entre 4 anos e 8 meses e 7 anos e 3 meses, cinco do gênero feminino e cinco do masculino, atendidos em projeto de pesquisa sobre terapia fonoaudiológica para casos de desvio fonológico. O projeto foi aprovado pelo Comitê de Ética e Pesquisa (CEP) da UFSM, sob número 108/05 e, anteriormente à participação dos sujeitos na pesquisa, seus pais ou responsáveis assinaram Termo de Consentimento Livre e Esclarecido autorizando a participação e posterior publicação dos resultados.

Para integrar o banco de dados do projeto os sujeitos deveriam apresentar os seguintes critérios: trocas na fala; idade acima dos 4 anos; audição normal para fala; estruturas e habilidades motoras orais normais; não ter feito terapia fonoaudiológica anterior; ser membro de uma família de falantes monolíngues do português brasileiro; ausência de problemas neurológicos relevantes para a produção da fala; capacidades intelectuais adequadas para o desenvolvimento da linguagem falada; compreensão da linguagem falada apropriada à idade mental; capacidades de linguagem expressiva aparentemente bem desenvolvidas, em termos de abrangência de vocabulário e comprimento dos enunciados.

A fim de verificar os critérios acima descritos, que definem o diagnóstico de desvio fonológico, todos os sujeitos foram submetidos às avaliações fonoaudiológicas: linguagem compreensiva e expressiva, sistema estomatognático, discriminação auditiva, consciência fonológica, exame articulatório e avaliação fonológica. Os resultados das avaliações encontravam-se dentro dos padrões de normalidade para cada faixa etária, com exceção da avaliação fonológica. Também foram realizadas avaliações complementares (audiológica, otorrinolaringológica e neurológica) com o intuito de descartar qualquer prejuízo orgânico ou funcional.

A avaliação da fala foi realizada por meio do instrumento Avaliação Fonológica da Criança - $\mathrm{AFC}^{(15)}$, a partir do qual os dados foram coletados em atividade de nomeação espontânea de figuras, gravados e posteriormente transcritos. Após a transcrição foi realizada análise contrastiva e por traços distintivos. Com a análise contrastiva obteve-se o inventário fonético e o sistema fonológico de cada sujeito. Cada som foi considerado presente no inventário fonético quando ocorreu duas vezes ou mais, independentemente de sua posição na palavra ${ }^{(16)}$. Para a obtenção do sistema fonológico, considerou-se um segmento adquirido, quando havia de $80 \%$ a $100 \%$ de produções corretas; parcialmente adquirido de $40 \%$ a $79 \%$ e não-adquirido, de $0 \%$ a $39 \%$.

Os sujeitos selecionados para a presente pesquisa deveriam ter recebido tratamento através do Modelo de Oposições Múltiplas $^{(4)}$ ou do Modelo ABAB-Retirada e Prova Múltiplas ${ }^{(5)}$. As crianças tratadas pelo Modelo de Oposições Múltiplas deveriam apresentar sistema fonológico compatível com o modelo proposto ${ }^{(4)}$, ou seja, substituição de muitos sons para um mesmo som; e aquelas tratadas pelo Modelo ABAB-Retirada e Provas Múltiplas deveriam ter completado 3 ciclos de tratamento.

A amostra foi distribuída em dois grupos. O grupo 1 (G1) era composto por sujeitos tratados pelo Modelo de Oposições Múltiplas ${ }^{(4)} \mathrm{em}$ que são selecionados os sons que a criança substitui por um único fonema (por exemplo: os fonemas $/ \mathrm{R} /, / \mathrm{k} /$, $/ \mathrm{g} / \mathrm{e} / \mathrm{m} /$ são substituídos por [s]). A terapia envolve um grupo de palavras que diferem em apenas um fonema, por exemplo: rola x mola x cola $\mathrm{x}$ gola $\mathrm{x}$ sola, que quando produzidas pela criança, gera homônimos. Geralmente este modelo é aplicado em crianças com desvios fonológicos mais graves, pois nesses casos as substituições de vários sons por um único som são mais frequentes nestas gravidades, não sendo tão comuns em desvios mais leves; isto é, nem todas as crianças poderão ser tratadas com este modelo. O grupo 2 (G2), por sua vez, era composto por sujeitos tratados pelo Modelo ABAB-Retirada e Provas Múltiplas ${ }^{(5)}$ em que apenas um som-alvo é selecionado a partir dos traços distintivos. Este modelo terapêutico baseia-se no pressuposto de que um som é tratado considerando-se a hierarquia implicacional dos traços distintivos, em que no tratamento de sons de maior complexidade haverá a aquisição de sons menos complexos, sem intervenção direta sobre os mesmos. Este modelo pode ser aplicado no tratamento de casos de desvios mais leves, com gravidade moderado-grave, levemente-moderada e leve.

O G1 foi composto por S1, S2, S3, S4 e S5 e o G2 por S6, S7, S8, S9 e S10. Os sujeitos foram pareados (S1 e S6; S2 e S7; S3 e S8; S4 e S9; S5 e S10) quanto ao número de fonemas não adquiridos no sistema fonológico, pré-tratamento, a idade e ao gênero. A média de fonemas não adquiridos em ambos os grupos foi de sete fonemas. Em relação à idade, a média foi de 5 anos e 11 meses para o G1 e 5 anos e 6 meses para G2. Quanto ao gênero, em cada um dos grupos havia três meninos e duas meninas.

A terapia recebida pelos sujeitos do G1, modelo de Oposições Múltiplas, constou de 25 sessões, exceto para uma criança que, após 15 sessões tinha apenas um som ausente no sistema fonológico, impossibilitando a continuidade ao modelo proposto.

Nas sessões com o modelo de Oposições Múltiplas, foi utilizada a estrutura terapêutica proposta em estudo anterior ${ }^{(18)}$ em que, antes de iniciar o tratamento, realiza-se a linha de base, sondagem realizada antes do início da terapia onde cada som não-adquirido e parcialmente adquirido no sistema fonológico é testado (este sistema é obtido por meio da avaliação fonológica pré-tratamento). Para realizá-la, são selecionadas 
seis palavras que exijam cada um dos fonemas ausentes, e que possam ser representadas por figuras, para a criança nomear. Esta mesma linha de base, denominada posteriormente de sondagem, foi utilizada no decorrer das sessões a fim de monitorar as evoluções dos pacientes.

Após a determinação da linha de base, iniciou-se o tratamento propriamente dito. Foram duas sessões semanais de 45 minutos cada, totalizando 30 sessões de terapia, com uma sondagem a cada cinco sessões. Após o término das sessões, foi realizada novamente a avaliação fonológica. A sessão terapêutica foi sempre iniciada e finalizada com o bombardeio auditivo, para o qual eram selecionadas quatro palavras-alvo para cada som-alvo selecionado.

A terapia do G2, submetido ao Modelo ABAB-Retirada e Provas Múltiplas, constou de 27 sessões (duas sessões semanais de 45 minutos cada). Quanto à estrutura terapêutica adotada, iniciou-se pela coleta dos dados da fala (A1), realizada mediante gravação da nomeação e fala espontânea, seguida pela transcrição e análise fonológica. Em seguida, foram determinados os traços distintivos alterados e, a partir disto, delimitado o som-alvo para o tratamento, bem como as respectivas palavras-alvo e as do bombardeio auditivo. Neste Modelo seleciona-se apenas um som-alvo para ser tratado durante um ciclo (nove sessões) ou mais. O bombardeio auditivo era composto por 15 palavras selecionadas, cuidadosamente contendo o som-alvo do tratamento. A criança apenas escutava e observava as produções do terapeuta, não havendo necessidade de repetir. A lista de palavras era lida no início e no final das sessões.

A intervenção terapêutica, no primeiro ciclo de tratamento (B1), teve duração de nove sessões. Durante o ciclo foram realizadas três Provas-Alvo Básicas (PAB), na primeira, quinta e nona sessões, as quais avaliaram a aquisição do som-alvo nas palavras alvo e nas não-alvo. Ao término do tratamento (B1) foram realizadas cinco sessões do Período de Retirada (A2), que consistiu de um intervalo sem tratamento direto sobre o som-alvo. Nestas sessões foram realizadas coletas da nomeação e fala espontânea a fim de observar as generalizações obtidas quanto à aquisição de sons-alvo e não-alvo no sistema fonológico destes sujeitos.

Para a análise dos resultados foi comparada a diferença do número de sons ausentes no sistema fonológico e a média do percentual de ocorrência de generalizações (dentro de uma classe de sons e para outras classes) pré e pós-tratamento, entre G1 e G2 a partir do teste t de Student para duas amostras independentes, considerando-se $\mathrm{p}<0,05$. Além disso, foi realizada análise qualitativa das generalizações a partir dos dados obtidos pré e pós-tratamento.

\section{RESULTADOS}

A Figura 1 ilustra a média de sons ausentes entre os grupos. Observa-se que o G1 apresentou menor média $(5,6)$ que o G2 $(6,6)$, ou seja, as crianças do G1 adquiriram maior número de sons no seu sistema fonológico, contudo esta diferença não foi significativa.

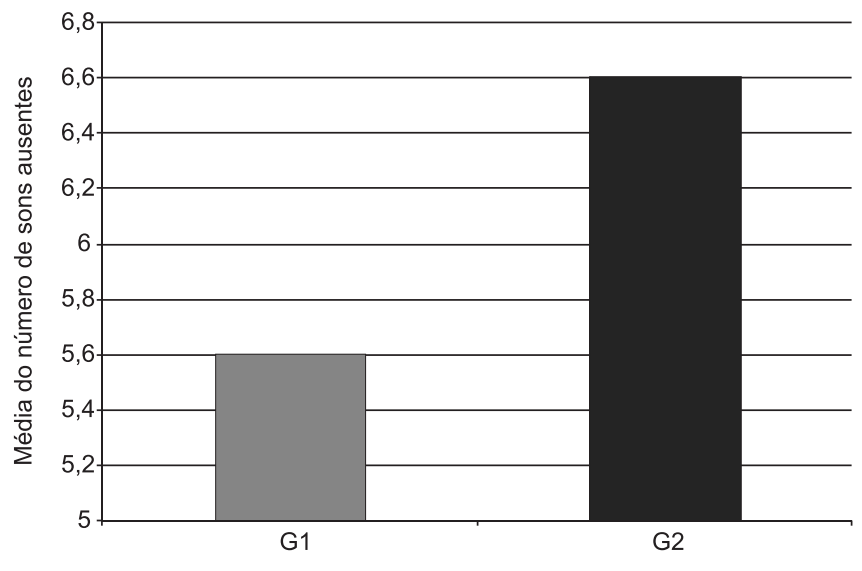

Legenda: G1 = grupo 1, tratado pelo modelo de Oposições Múltiplas; G2 = grupo 2, tratado pelo modelo ABAB-Retirada e Provas Múltiplas Nota: Análise estatística por meio do teste t de Student, para duas amostras independentes, obteve $p=0,3283$

Figura 1. Média do número de sons ausentes entre os grupos

A Tabela 1 representa a comparação da generalização dentro da classe de sons e para outras classes de sons. Em relação à generalização dentro da classe de sons, verifica-se que o G1 apresentou maiores mudanças, entretanto a diferença também não foi significativa. Também não houve diferença na generalização para outras classes de sons, embora ela tenha ocorrido mais no $\mathrm{G} 2$.

Na Tabela 2 estão representados os tipos de generalização estrutural apresentados pelas crianças, na avaliação inicial e final, no tratamento pelos Modelos ABAB-Retirada e Provas Múltiplas e de Oposições Múltiplas.

Verifica-se a ocorrência dos dois tipos de generalização para todos os sujeitos exceto o S1, que apresentou regressões durante o tratamento. Observa-se, também que os maiores diferenciais da generalização dentro de uma classe de sons ocorreram principalmente no G1, tratado pelo modelo de Oposições Múltiplas, enquanto que a generalização para outras classes de sons ocorreu no G2, tratados pelo ABAB-Retirada e Provas Múltiplas.

Tabela 1. Comparação da generalização entre os grupos

\begin{tabular}{|c|c|c|c|c|}
\hline & \multicolumn{2}{|c|}{ Generalização dentro da classe de sons } & \multicolumn{2}{|c|}{ Generalização para outras classes de sons } \\
\hline & Média (\%) & $\mathrm{DP}(\%)$ & Média (\%) & $\mathrm{DP}(\%)$ \\
\hline G1 & 47,97 & 13,44 & 24,08 & 9,54 \\
\hline G2 & 28,12 & 9,34 & 29,57 & 14,49 \\
\hline Valor de $p$ & \multicolumn{2}{|c|}{0,1300} & \multicolumn{2}{|c|}{0,3798} \\
\hline
\end{tabular}

Teste t de Student, para duas amostras independentes $(p<0,05)$

Legenda: G1 = grupo 1, tratado pelo modelo de Oposições Múltiplas; G2 = grupo 2, tratado pelo modelo ABAB-Retirada e Provas Múltiplas; DP = desvio-padrão 
Tabela 2. Tipos de generalização obtida pré e pós-tratamento

\begin{tabular}{|c|c|c|c|c|c|c|c|}
\hline \multirow{3}{*}{ Modelo } & \multirow{3}{*}{ Sujeito } & \multicolumn{6}{|c|}{ Tipo de generalização (\%) } \\
\hline & & \multicolumn{3}{|c|}{ Dentro de uma classe de sons } & \multicolumn{3}{|c|}{ Outras classes de sons } \\
\hline & & $\mathrm{Al}$ & AF & Dif & $\mathrm{Al}$ & AF & Dif \\
\hline \multirow{5}{*}{ Oposições Múltiplas } & S1 & 76,2 & 52,9 & 0,0 & 33,3 & 40,8 & 7,5 \\
\hline & S2 & 50,0 & 81,8 & 31,8 & 42,4 & 67,8 & 25,4 \\
\hline & S3 & 0,0 & 57,1 & 57,1 & 0,0 & 22,9 & 22,9 \\
\hline & S4 & 45,1 & 100,0 & 54,9 & 36,7 & 100,0 & 63,3 \\
\hline & S5 & 1,0 & 28,2 & 27,2 & 49,4 & 70,8 & 21,4 \\
\hline \multirow{5}{*}{ ABAB-Retirada e Provas Múltiplas } & S6 & 0,0 & 15,6 & 15,6 & 27,0 & 81,9 & 54,9 \\
\hline & S7 & 0,0 & 7,0 & 7,0 & 22,5 & 91,1 & 68,6 \\
\hline & S8 & 38,1 & 48,8 & 10,7 & 17,4 & 100,0 & 82,6 \\
\hline & s9 & 9,5 & 37,7 & 28,2 & 37,8 & 56,4 & 18,6 \\
\hline & $\mathrm{S} 10$ & 21,8 & 81,5 & 59,7 & 9,0 & 24,2 & 15,2 \\
\hline
\end{tabular}

Legenda: $\mathrm{Al}$ = avaliação inicial; $\mathrm{AF}$ = avaliação final; Dif = diferencial

\section{DISCUSSÃO}

Ambos os modelos estudados, Oposições Múltiplas e ABAB-Retirada e Provas Múltiplas, favoreceram aquisições no sistema fonológico das crianças bem como os diferentes tipos de generalização (dentro de uma classe e outras classes de sons). Resultados semelhantes foram verificados em outros estudos ${ }^{(12-14,19,20)}$ os quais verificaram a eficácia desses modelos terapêuticos. O modelo de Oposições Múltiplas obteve maior média de fonemas adquiridos do que o modelo ABAB-Retirada e Provas Múltiplas. Esses resultados podem estar relacionados com o número de fonemas selecionados para tratamento (mais de três).

A generalização dentro de uma classe de sons foi mais evidente no modelo de Oposições Múltiplas, provavelmente porque neste modelo são contrastados inúmeros fonemas em relação ao substituto e geralmente os sons selecionados pertencem a diferentes classes de sons ${ }^{(4,21)}$. Logo, o fato de sons de diferentes classes serem selecionados pode ter favorecido este tipo de generalização, uma vez que em cada classe existe uma gama de fonemas a serem adquiridos.

O S1, submetido ao modelo de Oposições Múltiplas, apresentou percentual de generalização menor na avaliação final, em comparação com a avaliação inicial. Embora sem significância estatística, tais achados podem estar relacionados com o processo de regressão, comum em crianças que estão reorganizando seu sistema fonológico ${ }^{(22)}$.

A generalização para outras classes de sons foi maior, embora também sem diferença estatística, nas crianças tratadas pelo modelo ABAB-Retirada e Provas Múltiplas. Considera-se que neste modelo, embora tenha sido selecionado apenas um som para tratamento, este apresentava alto nível de com- plexidade, ou seja, era um som mais complexo na hierarquia implicacional. Isso confirma que, quanto maior o nível de complexidade no tratamento, maiores serão as generalizações a níveis menos complexos, o que significa que, tratando sons mais difíceis, sons mais fáceis podem ser adquiridos sem tratamento direto ${ }^{(16)}$. Além disso, pelo fato deste modelo utilizar como prerrogativa o tratamento de apenas um som, pertencente a uma única classe de sons, há maior possibilidade de ocorrer esse tipo de generalização. Resultados semelhantes foram encontrados em outra pesquisa ${ }^{(19)}$, em que as autoras verificaram a ocorrência deste tipo de generalização principalmente nas crianças submetidas ao ABAB-Retirada e Provas Múltiplas e Oposições Máximas.

\section{CONCLUSÃO}

Treinar sons individualmente ou treiná-los em conjunto são duas formas alternativas no tratamento do desvio fonológico, e isso dependerá do conhecimento prévio do sistema fonológico do caso a ser tratado. Conclui-se que ambos os métodos (ABAB-Retirada e Provas Múltiplas e Oposições Máximas) favoreceram mudanças no sistema fonológico das crianças estudadas, proporcionando a aquisição de fonemas e a ocorrência de generalizações tanto para outras classes de sons como dentro de uma mesma classe de sons. Logo, os achados desta pesquisa apontam não haver diferença na efetividade do tratamento quando os sons são tratados isoladamente ou em conjunto. Entretanto, há uma limitação importante quanto ao número de sujeitos pesquisados, o que não permite a ampla generalização destes achados. O mesmo ocorre quanto à seleção e utilização dos modelos de terapia, que parecem ser mais indicados para sistemas fonológicos específicos. 


\begin{abstract}
Purpose: To verify whether the stimulation of groups of sounds results in greater phonological changes than the stimulation of single sounds, and to analyze the occurrence of generalization across and within sound classes. Methods: The sample comprised ten children with phonological disorders, with ages between 4 years and 8 months and 7 years and 3 months, divided into two groups. Group 1 (G1) was treated by the Multiple Oppositions approach, and Group 2 (G2), by the ABAB-Withdrawal and Multiple Probes approach. The treatment of G1 consisted of stimulation of the contrast of many sounds presented together, while the treatment of G2 involved stimulation of only one target sound per cycle of treatment. It was analyzed the number of absent sounds and the occurrence of generalization across and within sound classes, pre- and post-treatment. Data were statistically and descriptively analyzed. Results: G1 acquired more sounds than G2; however, this difference was not significant. Considering the generalization within sound class, G1 presented major changes, while generalization across sound classes was greater for G2, although none of these results were statistically significant. Conclusion: Both methods favored changes in the phonological system of the children studied, allowing the acquisition of phonemes and the occurrence of generalizations. There is no difference between them regarding treatment effectiveness.
\end{abstract}

Keywords: Speech disorders; Speech; Speech therapy; Generalization, response; Sound; Child

\title{
REFERÊNCIAS
}

1. Weiner F. Treatment of phonological disability using the method of meaningful minimal contrast: two case studies. J Speech Hear Disord. 1981;46(1):97-103.

2. Gierut JA. Maximal opposition approach to phonological treatment. J Speech Hear Disord. 1989;54(1):9-19.

3. Gierut JA. The conditions and course of clinically-induced phonological change. J Speech Lang Hear Res. 1992; 35(5):1049-63.

4. Williams AL. Multiple oppositions: theoretical foundations for an alternative contrastive intervention framework. Am J Speech-Lang Pathol. 2000;9:282-8.

5. Tyler AA, Figurski GR. Phonetic inventory changes after treating distinctions along an implicational hierarchy. Clin Linguist Phon. 1994;8(2):91-107.

6. Dean E, Howell J. Developing linguistic awareness: a theoretically based approach to phonological disorders. Br J Disord Commun. 1986;21(2):223-38.

7. Tyler AA, Edwards ML, Saxman JH. Acoustic validation of phonological knowledge and its relationship to treatment. J Speech Hear Disord. 1990;55(2):251-61.

8. Hesketh A, Adams C, Nightingale C, Hall R. Phonological awareness therapy and articulatory training approaches for children with phonological disorders: a comparative outcome study. Int J Lang Commun Dis. 2000;35(3):337-54.

9. Barberena LS, Keske-Soares M, Mota HB. Generalização baseada nas relações implicacionais obtida pelo modelo "ABAB-Retirada e Provas Múltiplas”. Rev Soc Bras Fonoaudiol. 2008;13(2):143-53.

10. Ardenghi LG, Mota HB, Keske-Soares M. A terapia Metaphon em casos de desvios fonológicos. Rev Soc Bras Fonoaudiol. 2006;11(2):106-15.

11. Keske-Soares M, Donicht G, Chacalin MA, Ghisleni MR. Generalização por 'reforço' ou 'contraste' no tratamento do desvio fonológico. Rev Soc Bras Fonoaudiol. 2008;13(4):391-7.

12. Mota HB, Keske-Soares M, Bagetti T, Ceron MI, Melo Filha MG. Análise comparativa da eficiência de três modelos de terapia fonológica. Pró-Fono. 2007;19(1):67-74.
13. Keske-Soares M, Brancalioni AR, Marini C, Pagliarin KC, Ceron MI. Eficácia da terapia para desvios fonológicos com diferentes modelos terapêuticos. Pró-Fono. 2008;20(3):153-8.

14. Pagliarin KC, Mota HB, Keske-Soares M. Análise da eficácia terapêutica em três modelos fonológicos de abordagem contrastiva. Pró-Fono. 2009; 21(4):297-302.

15. Yavas M, Hernandorena CL, Lamprecht RR. Avaliação fonológica da criança: reeducação e terapia. Porto Alegre: Artes Médicas; 2001.

16. Keske-Soares M. Terapia fonoaudiológica fundamentada na hierarquia implicacional dos traços distintivos aplicada em crianças com desvios fonológicos. [tese]. Porto Alegre: Pontifícia Universidade Católica do Rio Grande do Sul; 2001.

17. Bernhardt B. The application of nonlinear phonological theory to intervention with one phonologically disorders child. Clin Linguist Phon. 1992;6(4):283-316.

18. Bagetti T, Mota HB, Keske-Soares M. Modelo de oposições máximas modificado: uma proposta de tratamento para o desvio fonológico. Rev Soc Bras Fonoaudiol. 2005;10(1):36-42.

19. Ceron M, Keske-Soares M. Terapia fonológica: a generalização a itens não utilizados no tratamento (outras palavras). Rev CEFAC. 2007;9(4):453-60.

20. Ceron M, Keske-Soares, M. Terapia fonológica: a generalização dentro de uma classe de sons e para outras classes de sons. Rev CEFAC. 2008;10(3):311-20.

21. Williams AL. Assessment, target selection, and intervention: dynamic interactions within a systemic perspective. Top Lang Disord. 2005;25(3):231-42.

22. Strauss S. U-shaped behavioral growth. New York: Academic Press; 1982. 